\title{
Investigación numérica de las condiciones de lubricación en chumaceras hidrodinámicas con el efecto del desalineamiento del eje
}

\section{Numerical Investigations of the Lubrication Conditions in Hydrodynamic Bearings with Shaft Misalignment Effect}

\author{
Antonio-García A. \\ Instituto de Electrónica y Mecatrónica \\ Universidad Tecnológica de la Mixteca \\ Huajuapan de León, Oaxaca \\ Correo:agarcia@mixteco.utm.mx \\ Linares-Flores J. \\ Instituto de Electrónica y Mecatrónica \\ Universidad Tecnológica de la Mixteca \\ Huajuapan de León, Oaxaca \\ Correo:jlinares@mixteco.utm.mx
}

\author{
Arias-Montiel M. \\ Instituto de Electrónica y Mecatrónica \\ Universidad Tecnológica de la Mixteca \\ Huajuapan de León, Oaxaca \\ Correo:mam@mixteco.utm.mx
}

Información del artículo: recibido: agosto de 2011, aceptado: mayo de 2012

\section{Resumen}

Las chumaceras hidrodinámicas radiales se usan ampliamente para soportar carga en máquinas rotatorias que giran a alta velocidad. Teóricamente, las chumaceras hidrodinámicas se diseñan para una vida infinita, pero la precisión en el maquinado, el calentamiento o el desalineamiento con el rotor pueden contribuir a problemas de funcionamiento y a un desgaste indeseado. Un problema que no se puede eliminar es el desalineamiento angular del eje provocado por el peso y la flexibilidad del rotor. El desalineamiento cambia el espesor de película del lubricante afectando las condiciones de lubricación hidrodinámica. En este trabajo se presenta una investigación numérica del comportamiento del campo de presión, el flujo axial, el espesor de película y la fricción viscosa, considerando el desalineamiento del eje por el efecto del peso y la flexibilidad del rotor. El campo de presión hidrodinámica se resuelve utilizando la ecuación de la lubricación de Reynolds junto con una expresión modificada del espesor de película que incluye el desalineamiento del eje. También se presenta una expresión para calcular el máximo desalineamiento permitido por una chumacera, en función del punto de equilibrio de estado estable. Los resultados muestran que a medida que el desalineamiento del eje se incrementa el espesor de película se reduce, causando el incremento de la presión máxima y del flujo axial del lubricante, así como pequeños cambios en la fricción viscosa. Con la finalidad de validar los resultados numéricos obtenidos en esta investigación, se compararon con resultados numéricos reportados en la literatura internacional, dando una buena aproximación.

\section{Descriptores:}

- chumaceras hidrodinámicas

- desalineamiento

- flujo axial

- espesor de película

- fricción viscosa 


\begin{abstract}
The radial hydrodynamic bearings are widely used to support loads in rotating machines that operate at high speed. Theoretically, hydrodynamic bearings are designed for infinite life, but the precision machining, heating or misalignment with the rotor can contribute to performance problems and undesired wear. A problem that cannot be eliminated is the shaft angular misalignment caused by the weight and flexibility of the rotor. Misalignment changes the lubricant film thickness affecting the conditions of hydrodynamic lubrication. In this paper, a numerical investigation of the behavior of the pressure field, axial flow, film thickness and viscous friction considering the misalignment of the shaft by the effect of the weight and flexibility of the rotor, is presented. The hydrodynamic pressure field is solved using the equation of Reynolds lubrication with a modified expression of film thickness including the shaft misalignment. An expression to calculate the maximum misalignment allowed by a bearing based on the equilibrium steady state is also presented. The results show that as the shaft misalignment increases, a reduction in the film thickness is present, causing an increase in both maximum pressure and axial flow of lubricant, and also small changes in viscous drag. In order to validate the numerical results obtained in this investigation they were compared with numerical results reported in international literature, giving a good approximation.
\end{abstract}

\section{Introducción}

Las chumaceras hidrodinámicas se usan principalmente en grandes máquinas rotatorias que giran a altas velocidades. Un buen diseño de la chumacera, suministro y viscosidad del fluido lubricante, permiten que durante la operación de la máquina se forme una cuña de lubricante, la cual separa al eje de la chumacera evitando el contacto entre metal y metal. La capacidad de carga que tienen las chumaceras se debe a la generación del campo de presión en la película de lubricante. Por medio de este campo de presión se puede evaluar si las dimensiones de la chumacera, el claro radial y el lubricante, mantendrán las condiciones adecuadas de lubricación hidrodinámica. Generalmente, la velocidad de giro y el peso del rotor son los datos iniciales a partir de los cuales se determinan la longitud, el diámetro, el claro radial, así como la viscosidad del lubricante. Los parámetros de diseño junto con las tablas y gráficas proporcionadas en la literatura (Raimondi y Boid, 1958) permiten conocer el valor de la presión máxima, el flujo axial, el espesor de película y la fricción viscosa, los cuales cambian con la velocidad de giro del rotor y son importantes para mantener las condiciones de lubricación hidrodinámica.

En las tablas y gráficas presentadas por Raimondi y Boid (1958) se considera que la distancia entre las líneas de centro del eje y de la chumacera es constante. Esta distancia se conoce como excentricidad e. En casos reales, las líneas de centro del eje y de la chumacera están sujetas a un cierto grado de desalineamiento angular. El des- alineamiento puede ser causado por diversos factores, por ejemplo, inexactitudes por un mal montaje, mal maquinado, el peso del rotor, la flexibilidad del eje, etcétera, aún si el montaje y el maquinado fuesen muy precisos, siempre existirá un cierto grado de desalineamiento debido a la flexibilidad del eje y al peso del rotor. En la figura 1 se considera una configuración simétrica (rotor de Jeffcott) de un sistema rotor-chumaceras, la cual muestra una rotación $\alpha_{x}$ del eje en un sistema de referencia $\left(y_{0}, z_{0}\right)$ ubicado en el centro de la chumacera. En este caso, el peso y la flexibilidad del eje provocan que las líneas de centro del eje y de la chumacera no coincidan, haciendo que el espesor de película sea función de la excentricidad del centro del muñón $e_{0} \mathrm{y}$ del ángulo de rotación $\alpha_{x}$ (figura 2). Para analizar las condiciones de lubricación en chumaceras hidrodinámicas es necesario calcular el ángulo $\alpha_{x}$ en cada chumacera, lo cual puede realizarse por medio de la teoría de vigas.

En la literatura internacional se han reportado diversos trabajos teóricos y experimentales sobre el tema del desalineamiento en chumaceras hidrodinámicas. McKee y McKee (1932) realizaron uno de los primeros trabajos experimentales, mostrando que el campo de presión se desarrolla en forma anti-simétrica en la dirección axial. Dubois et al., (1955) estudiaron experimentalmente el cambio en la excentricidad, el flujo axial y las temperaturas locales en chumaceras desalineadas. Galleti y Capriz (1965) hicieron un estudio analítico sobre el par producido en chumaceras hidrodinámicas cortas con desalineamiento. Las investigaciones realizadas por Nicholas (1972) muestran que 
las chumaceras hidrodinámicas presentan poca resistencia contra el desalineamiento. Nicholas (1972) estableció que la intensidad del par producido por el desalineamiento induce poca influencia sobre la excentricidad y la rapidez del flujo axial, pero que la presión máxima se incrementa significativamente. Mokhtar et al., (1985) hacen una evaluación de las características térmicas en chumaceras desalineadas usando un enfoque adiabático.

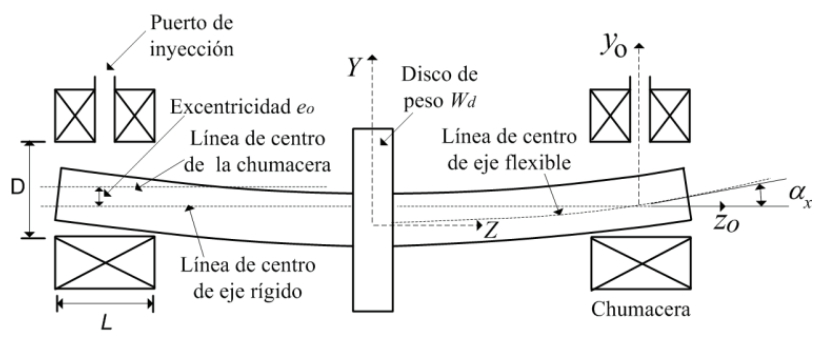

Figura 1. Rotor soportado por chumaceras hidrodinámicas

Buckholz y Lin (1986) analizaron el efecto del desalineamiento y la cavitación en chumaceras parciales lubricadas con fluidos No-Newtonianos. Qiu y Tieu (1996) desarrollaron un equipo experimental para medir el espesor de película, la presión y la temperatura en chumaceras desalineadas. Guha (2000) investigó las características de estado estable en chumaceras hidrodinámicas desalineadas considerando los efectos de la rugosidad isotrópica. Pierre et al. (2004) hicieron una investigación teórica y experimental de las condiciones de estado estable en chumaceras hidrodinámicas desalineadas, considerando un modelo termo-hidrodinámico. Recientemente, Jun et al., (2005) estudiaron las condiciones de lubricación considerando el desalineamiento en las chumaceras debido a la deformación por el peso del eje a una velocidad de operación fija. Jun et al., (2010) incluyen los efectos termo-hidrodinámicos y una superficie rugosa para el estudio de las condiciones de operación en chumaceras hidrodinámicas con desalineamiento.

En este trabajo se resuelve numéricamente la ecuación de la lubricación de Reynolds para diferentes valores de desalineamiento. Se presenta una expresión del espesor de película $h(\theta, z)$ que considera el ángulo de desalineamiento $\alpha_{x}$ causado por el peso $W_{d}$ y la flexibilidad del rotor. Para una determinada condición de operación se proporciona una expresión del rango de valores posibles para la rotación $\alpha_{x}$ en función de la $e_{0} \mathrm{y}$ el ángulo de attitud $\phi_{0}$. El campo de presión resultante se integra para obtener el flujo axial y el coeficiente de fricción viscosa del lubricante. También se obtienen el espesor de película mínimo y la presión máxima del lu- bricante. Los resultados se presentan en forma de gráficas adimensionales con la finalidad de que se utilicen en el diseño de chumaceras hidrodinámicas, en donde se considere el desalineamiento angular del eje. Para validar los resultados que se obtienen en esta investigación, se hace la comparación de la presión máxima $p_{\max }$ el flujo axial $Q_{a x i a l}$ el espesor de película mínimo $h_{\text {min }}$ y el coeficiente de fricción $F_{f} / W$ con un caso específico reportado en la literatura por Jun et al., (2005), obteniéndose una buena aproximación.

\section{Desarrollo}

Espesor de película

En la figura 2 se muestra una chumacera hidrodinámica con el muñón (eje en el interior) en una posición desalineada. Se consideran los sistemas de ejes fijos $X Y Z$ y $x y z$ en el centro de la chumacera y en el centro del muñón, respectivamente. La distancia del centro de la chumacera al centro del muñón se conoce como excentricidad $e_{0}$ y en el caso alineado es independiente de $z$. Se puede observar en la figura 2 que el desalineamiento causa una rotación $\alpha_{x}$ modificando al espesor de película $H(\tilde{\theta})$ del caso alineado a $H(\tilde{\theta}, z)$ para el caso desalineado. El espesor de película para el caso alineado está dado por (Childs, 1993; Szeri, 1998):

$H(\tilde{\theta})=C+e \cos (\tilde{\theta}-\phi)$

donde

$C=R-R_{1}$ es el claro radial (figura 3 ),

$\phi$ es el ángulo de attitud y

$\tilde{\theta}$ es la coordenada circular.

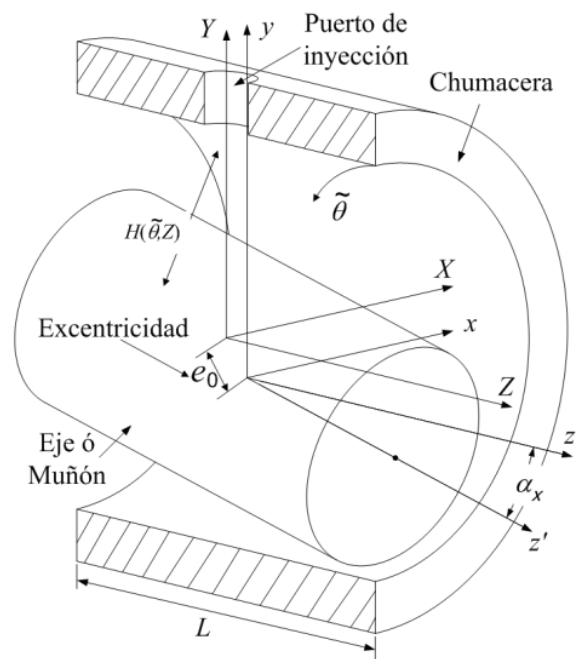

Figura 2. Vista en 3D de una chumacera desalineada 
Para obtener el espesor de película con desalineamiento que considere el peso y la flexibilidad del eje, es necesario tomar en cuenta el ángulo de rotación $\alpha_{x}$ del muñón. La relación entre la excentricidad $e$ a una distancia $z$ con la excentricidad del centro del muñón $e_{0}$ y el ángulo $\alpha_{x}$ se obtiene de la figura 3 como se indica a continuación:

$e \sin \phi=e_{0} \sin \phi_{0} e \cos \phi=e_{0} \cos \phi_{0}+\alpha_{x} z$

Si se sustituyen las ecuaciones (2) en (1) se obtiene la ecuación (3) del espesor de película que considera el desalineamiento angular $\alpha_{x}$ del muñón.

$H(\tilde{\theta}, z)=C+e_{0} \cos \left(\tilde{\theta}-\phi_{0}\right)+\alpha_{x} z \cos \tilde{\theta}$

En forma adimensional:

$h(\tilde{\theta}, z)=1+\varepsilon_{0} \cos \left(\tilde{\theta}-\phi_{0}\right)+\bar{z} \xi_{x} \cos \tilde{\theta}$

donde $h=H / C, \varepsilon_{0}=e_{0} / C, z=(L / 2) \quad \bar{z}, \xi x=(R / C)(L / D) \alpha_{x^{\prime}}$ $D=R / 2$ donde $e_{0}$ está comprendida entre $0 \leq e_{0} \leq C$ o en forma adimensional $0 \leq \varepsilon_{0} \leq 1$ y la excentricidad $\varepsilon$ a una distancia $z=L / 2$ tendrá como limites $\varepsilon_{0} \leq \varepsilon \leq 1$.

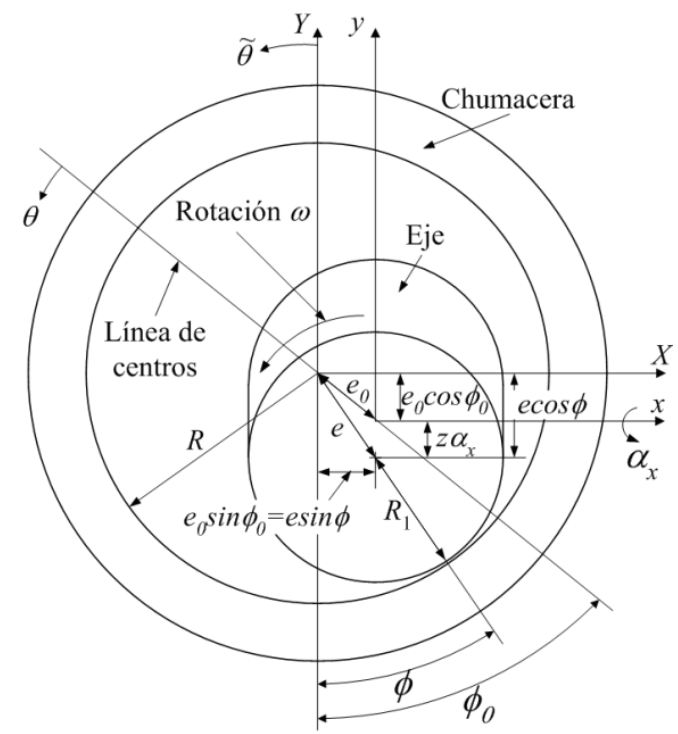

Figura 3. Vista frontal de una chumacera desalineada

Por medio de la teoría de vigas es posible calcular el ángulo $\alpha_{x}$ que el eje presenta en cada chumacera. Para una configuración de rotor de Jeffcott como la que se muestra en la figura 1, el ángulo $\alpha_{x}$ está dado por (Gere y Timoshenko, 1998):

$\alpha_{x}=\frac{W_{d} l^{2}}{16 E I}$ donde

$W_{d}$ es el peso del disco,

$l$ es la longitud del eje entre soportes,

$E$ es el modulo de Young e

I es el momento de inercia del eje.

Si se considera que $E$ e I son constantes, entonces el ángulo $\alpha_{x}$ dependerá del peso $W_{d}$ del rotor. El claro radial $C$ y la relación $L / D$ son limitantes para el valor máximo que pueda tomar el ángulo $\alpha_{x}$ lo que no se considera en la teoría de vigas (ecuación 5). Para calcular el máximo ángulo $\alpha_{x}$ permitido por la geometría de la chumacera se resuelven las ecuaciones (2) haciendo $e=C$ en $z=L / 2$, obteniendo:

$\alpha_{x \max }=\frac{2}{L}\left(\sqrt{C^{2}-e_{0}^{2} \sin ^{2} \phi_{0}}-e_{0} \cos \phi_{0}\right)$

o en forma adimensional

$\xi_{x \max }=\sqrt{1-\varepsilon_{0}^{2} \sin ^{2} \phi_{0}}-\varepsilon_{0} \cos \phi_{0}$

Dados $W_{d^{\prime}} l, E, I, C$ y la relación $L / D$, además de $e_{0}$ y $\phi_{0}$ de una posición de equilibrio, se procede a calcular el ángulo $\alpha_{x}$ por medio de la teoría de vigas y el ángulo $\alpha_{x \max }$ permitido por la geometría de la chumacera. Si $\alpha_{x}<\alpha_{x \max }$ entonces el ángulo $\alpha_{x}$ está dentro del valor permitido por la geometría de la chumacera. Por otra parte, si $\alpha_{x}>\alpha_{x \max }$ entonces sólo en la chumacera se considerará que $\alpha_{x}=\alpha_{x \max }$ conservándose los valores calculados por la teoría de vigas para el resto del eje. En resumen, el ángulo $\alpha_{x}$ calculado por la teoría de vigas no puede ser mayor que $\alpha_{x \max }$ que tiene como limitante la geometría de la chumacera.

\section{Campo de presión}

La ecuación adimensional de Reynolds que define el campo de presión de estado estable para chumaceras hidrodinámicas está dada por (Childs, 1993; Szeri, 1998):

$\frac{\partial}{\partial \tilde{\theta}}\left(h^{3} \frac{\partial \tilde{p}}{\partial \tilde{\theta}}\right)+\left(\frac{D}{L}\right)^{2} \frac{\partial}{\partial \tilde{z}}\left(h^{3} \frac{\partial \bar{p}}{\partial \bar{z}}\right)=12 \pi \frac{\partial h}{\partial \tilde{\theta}}$

donde

$p=\mu N(R / C)^{2} \bar{p}$,

$\mu$ es la viscosidad y

$N$ es la velocidad de rotación en rps (revoluciones por segundo).

Para obtener el campo de presión con desalineamiento es necesario resolver la ecuación (8) junto con la ecuación (4) considerando las condiciones de frontera: 
$p(\tilde{\theta}, z)=0 \quad$ para $z= \pm L / 2$

y las condiciones de frontera de Swift-Stieber:

$p=\frac{\partial p}{\partial \tilde{\theta}}=\frac{\partial p}{\partial z}=0$ para $\tilde{\theta}=\tilde{\theta}(z)_{c a v}$

La ecuación (9) considera la presión en los extremos de la chumacera tomando como referencia la presión atmosférica. La ecuación (10) considera la cavitación, de tal forma que, tanto la presión como sus gradientes normales a la curva de cavitación no existan en la "frontera de inicio de cavitación". Esta condición de frontera se implementa numéricamente haciendo cero cualquier presión negativa calculada previamente en cada iteración. Lund y Thomsen (1978) establecen que el perfil de presión calculado de esta manera satisface el requisito del gradiente de presión cero en la frontera de cavitación. Esta condición de frontera ha ganado amplia aceptación en chumaceras hidrodinámicas con cargas estacionarias. El conjunto de condiciones de frontera se completa con la presión en el puerto de inyección $p_{0}$ para asegurar la lubricación.

$p_{0}=0 \quad$ para $\quad \tilde{\theta}=\tilde{\theta}_{i n y}$

Para mantener el flujo, la presión clásica de inyección $P_{0}$ debe ser ligeramente mayor que la presión atmosférica.

\section{Fuerzas hidrodinámicas}

Las fuerzas hidrodinámicas se obtienen integrando el campo de presión sobre el área de la chumacera, como se muestra en la figura 4 :

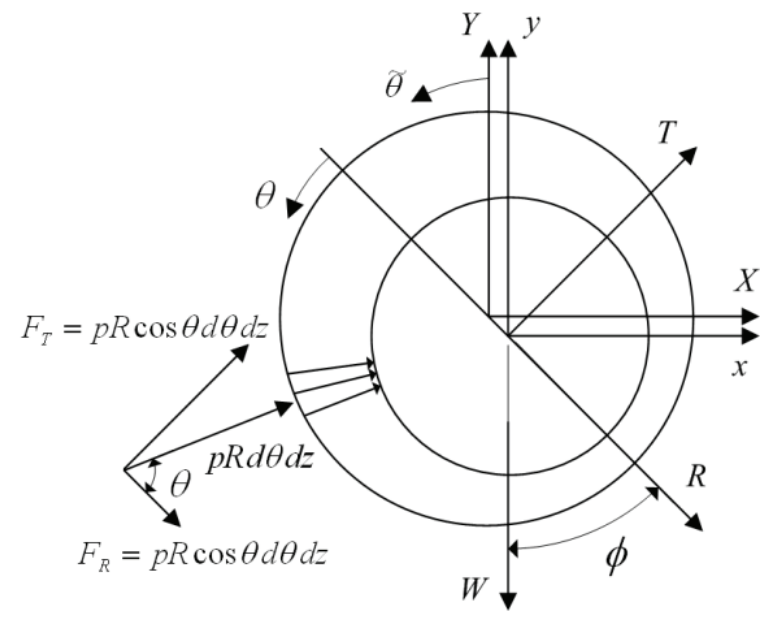

Figura 4. Fuerzas hidrodinámicas desarrolladas por el campo de presión
$\bar{F}_{X}=\frac{F_{X}}{\mu N L D(R / C)^{2}}=-\frac{1}{4} \int_{-1}^{1} \int_{\tilde{\theta}_{1}}^{\tilde{\theta} 2} \tilde{p} \sin \tilde{\theta} d \tilde{\theta} d \tilde{z}$

$\bar{F}_{Y}=\frac{F_{Y}}{\mu N L D(R / C)^{2}}=-\frac{1}{4} \int_{-1}^{1} \int_{\tilde{\theta}_{1}}^{\tilde{\theta}_{2}} \tilde{p} \cos \tilde{\theta} d \tilde{\theta} d \tilde{z}$

donde $\tilde{\theta}_{1}$ es la localización del puerto de inyección y $\tilde{\theta}_{2}$ es la localización de la curva de inicio de cavitación. Debido al desalineamiento, el ángulo $\tilde{\theta}_{2}$ cambia con respecto a la coordenada $z$ lo que no permite evaluar las fuerzas hidrodinámicas de manera analítica.

\section{Flujo axial}

El lubricante recircula en el interior de la chumacera y viaja hasta alcanzar la salida en $\pm L / 2$. El flujo de salida depende del campo de presión y del espesor de película (ecuación 14).

$q_{\text {axial }}=\frac{Q_{\text {axial }}}{N R L C}=\left.\frac{1}{12}\left(\frac{D}{L}\right)^{2} \int_{\tilde{\theta}_{1}}^{\tilde{\theta}_{2}} h^{3} \frac{\partial \bar{p}}{\partial \bar{z}}\right|_{ \pm L / 2} d \theta$

La cantidad de flujo axial deberá reponerse como flujo de entrada para mantener la lubricación hidrodinámica.

\section{Fricción viscosa}

La fuerza de fricción viscosa $F_{f}$ es la resistencia que se opone a la rotación del muñón debido al esfuerzo cortante del fluido, está dada por (Childs, 1993; Szeri, 1998):

$F_{f}=\int_{-L / 2}^{L / 2} \int_{0}^{2 \pi}\left(\frac{H}{2 R} \frac{\partial \bar{p}}{\partial \theta}+\frac{\mu \omega R}{H}\right) R d \theta d z$

o en forma adimensional:

$\left(\frac{R}{C}\right) f=\int_{-1}^{1} \int_{0}^{2 \pi}\left(\frac{S h}{8} \frac{\partial \bar{p}}{\partial \tilde{\theta}}+\frac{\pi S}{2 h}\right) d \tilde{\theta} d \bar{z}$

donde $f=F_{f} / W$ es el coeficiente de fricción, $S=\left(\bar{F}_{X}^{2}+\bar{F}_{Y}^{2}\right)^{-1 / 2}$ es el número de Sommerfeld, el cual es adimensional y está relacionado con la geometría, las propiedades y la carga que soporta la chumacera $\left(S=\mu N L D(R / C)^{2} / W\right)$. El número de Sommerfeld es muy utilizado en el diseño de chumaceras hidrodinámicas, ya que reúne paráme- 
tros geométricos, de operación y la viscosidad del lubricante. A continuación se describen los pasos utilizados en este trabajo para resolver numéricamente la ecuación de Reynolds y obtener las condiciones de lubricación hidrodinámica.

1) Para valores de $\varepsilon_{0}, L / D$ y $\xi_{x}$ (datos de entrada) se propone un valor para el ángulo de attitud $\phi_{0}$.

2) Se calcula el espesor de película $h(\tilde{\theta}, \bar{z})$ utilizando la ecuación (4).

3) La ecuación de Reynolds (8) se resuelve por diferencias finitas considerando las condiciones de frontera (9), (10) y (11). Para acelerar la convergencia se utiliza un coeficiente de sobre-relajación de 1.9.

4) Se evalúan las fuerzas hidrodinámicas $F_{X} \mathrm{y} F_{\gamma}$. En el caso de estado estable $F_{X}$ debe ser igual a cero y $F_{Y}$ igual a la carga $W,\left(W=W_{d} / 2\right)$.

5) Si $F_{X} \neq 0$ se propone un nuevo ángulo $\phi_{0}$ y se repite a partir del paso 2 .

6) Una vez que $F_{X}=0$ finaliza el cálculo del campo de presión.

7) Una vez conocido el campo de presión, se integran las ecuaciones (14) y (16) para obtener el flujo axial y la fricción viscosa.

\section{Resultados y discusión}

Con fines de validación, se hace la comparación de $h_{\text {min' }} p_{\text {max }}, Q_{\text {axial }}$ y $F_{f} / W$ que se obtienen en este trabajo, y los resultados reportados en la literatura por Jun et al. (2005), donde se considera el desalineamiento del eje. También se hace la comparación con resultados sin desalineamiento (Khonsari y Booser, 2001) con la finalidad de mostrar el cambio que sufren las condiciones de lubricación con el desalineamiento. Jun et al. (2005) presentan resultados numéricos (validados experimentalmente) de las condiciones de lubricación de un sistema rotor-chumaceras de Jeffcott para valores de carga $W_{d}$ de 200, 400,600,800, 1000 y $1200 \mathrm{~N}$ a una velocidad de operación fija de 1400 RPM. Los parámetros de la chumacera, del eje, las propiedades del lubricante y las condiciones de operación que utilizaron Jun et al. (2005) son: $R=25 \mathrm{~mm}, L=40 \mathrm{~mm}, C=0.07$ $\mathrm{mm}, \mu=0.0686$ Pa s, $l=1000 \mathrm{~mm}, d_{e j e}=40 \mathrm{~mm}$ de acero AISI 1045.

Para utilizar el algoritmo presentado en este trabajo es necesario calcular el ángulo $\xi_{x}$ para cada una de las cargas $W_{d}$ utilizando la teoría de vigas, verificando que $\xi_{x}<\xi_{x \max }$. Posteriormente, se proponen valores de excentricidad del centro del muñón $\varepsilon_{0}$ hasta que el número de Sommerfeld $S=\mu N L D(R / C)^{2} / W$ coincide con el calculado con los datos proporcionados por Jun et al. (2005).
Las figuras 5, 6, 7 y 8 muestran los resultados de la comparación de las condiciones de lubricación.

La figura 5 muestra que entre los resultados de $h_{\min }$ de esta investigación y los reportados por Jun et al. (2005) existe una buena aproximación. La gráfica permite ver que el incremento de $W_{d}$ causa que $h_{\text {min }}$ disminuya, tanto para el caso alineado como para el caso desalineado. Para el caso alineado $h_{\text {min }}=\mathrm{C}\left(1-\varepsilon_{0}\right)$ (Khonsari, 2001). Haciendo la comparación de $h_{\text {min }}$ entre el caso alineado y desalineado se tiene que para $W_{d}=200$ $\mathrm{N}, h_{\min }=0.0667 \mathrm{~mm}$ (caso alineado) y $h_{\min }=0.0594 \mathrm{~mm}$ (caso desalineado de este trabajo) existiendo una diferencia de $0.0073 \mathrm{~mm}$. Para $W_{d}=1200 \mathrm{~N}, h_{\min }=0.057 \mathrm{~mm}$ (caso alineado) y $h_{\min }=0.0058 \mathrm{~mm}$ (caso desalineado), siendo la diferencia de $0.0512 \mathrm{~mm}$. El segundo caso muestra claramente la importancia de considerar el desalineamiento del muñón en el diseño de chumaceras hidrodinámicas. El valor adecuado de $h_{\text {min }}$ dependerá de la rugosidad de los materiales para evitar el contacto entre metal y metal.

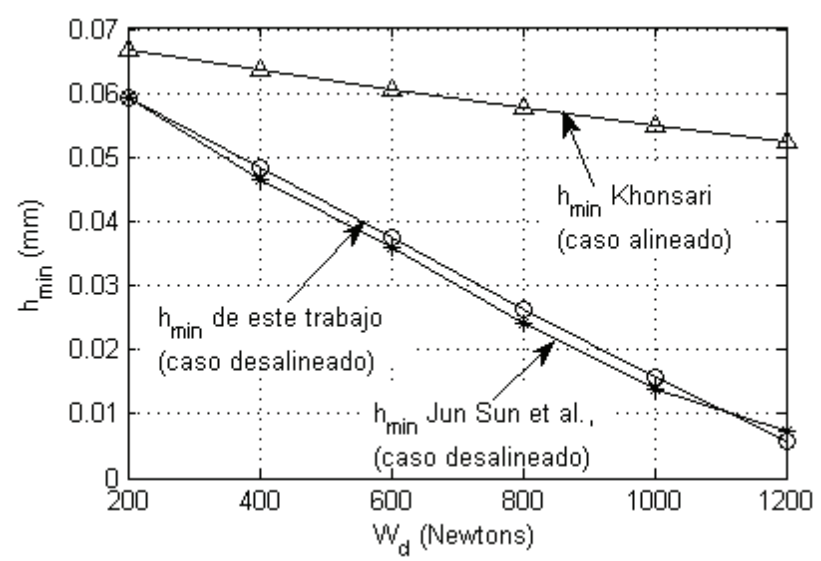

Figura 5. Comparación de $h_{\min }$ con y sin desalineamiento del eje

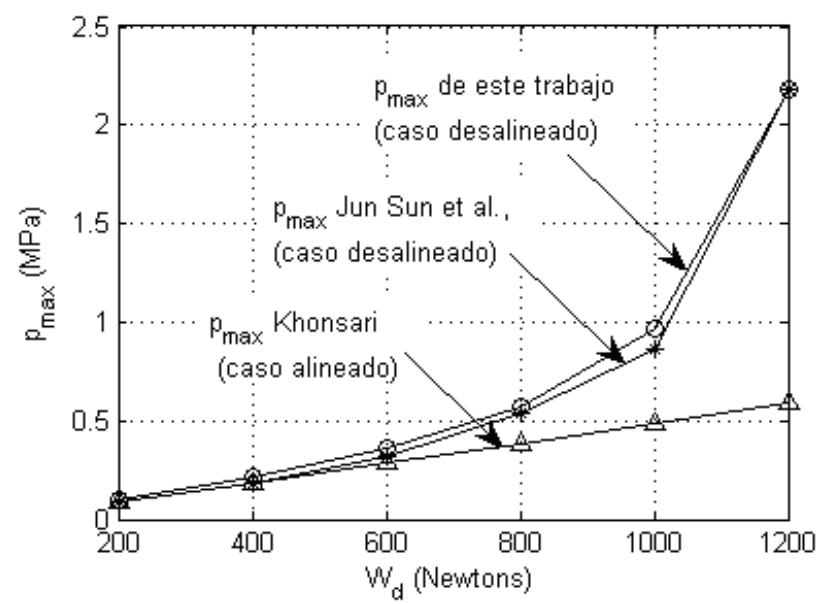

Figura 6. Comparación de $p_{\max }$ con y sin desalineamiento del eje 
La figura 6 muestra que la comparación entre la $p_{\max }$ de este trabajo y la reportada en la literatura tienen una buena aproximación. Sin considerar el desalineamiento, se tiene para $W_{d}=200 \mathrm{~N}$ una $p_{\max }=0.09236 \mathrm{MPa} \mathrm{y}$ para $W_{d}=1200 \mathrm{~N}$ una $p_{\max }=0.59 \mathrm{MPa}$, (Khonsari y Booser, 2001). La figura 6 muestra, para el caso desalineado (con resultados de este trabajo), una $p_{\max }=0.099 \mathrm{MPa}$ para $W_{d}=200 \mathrm{~N}$ y una $p_{\max }=2.1847 \mathrm{MPa}$ para $W_{d}=1200$ $\mathrm{N}$. Existiendo un incremento en la $p_{\max }$ de 1.072 veces para $W_{d}=200 \mathrm{~N}$ y de 3.7 veces para $W_{d}=1200 \mathrm{~N}$. El incremento en la presión máxima es una consecuencia de la reducción del espesor de película mínimo.

Es posible ver de las figuras 5 y 6 que el incremento de $W_{d}$ causa una disminución en $h_{\min } \mathrm{y}$ un incremento en $p_{\max }$, tanto en el caso alineado como en el desalineado, siendo el caso desalineado el que presenta cambios considerables tanto en $h_{\min }$ como en $p_{\max }$. Por otra parte, el incremento de $p_{\max }$ y la disminución de $h_{\min }$ causan un incremento en $Q_{\text {axial }}$ como se observa en la figura 7. Se puede ver que existe una buena aproximación entre los valores de $Q_{\text {axial }}$ con desalineamiento reportados en la literatura y los valores obtenidos en este trabajo. También se puede ver la diferencia entre los resultados de $Q_{\text {axial }}$ con y sin desalineamiento. Por ejemplo, para $W_{d}=200 \mathrm{~N}$ se tiene que $Q_{\text {axial }}=0.4246 \times 10^{-6} \mathrm{~m}^{3} / \mathrm{s}$ (caso alineado) y $Q_{\text {axial }}=0.6857 \times 10^{-6} \mathrm{~m}^{3} / \mathrm{s}$ (caso desalineado) obteniéndose un incremento de 1.614 en $Q_{\text {axial }}$. Se puede ver que a medida que la carga $W_{d}$ se incrementa, también aumenta la diferencia entre la $Q_{\text {axial }}$ del caso alineado y el caso desalineado. La importancia de conocer $Q_{\text {axial }}$ radica en que esta cantidad de lubricante debe reponerse para mantener la lubricación hidrodinámica.

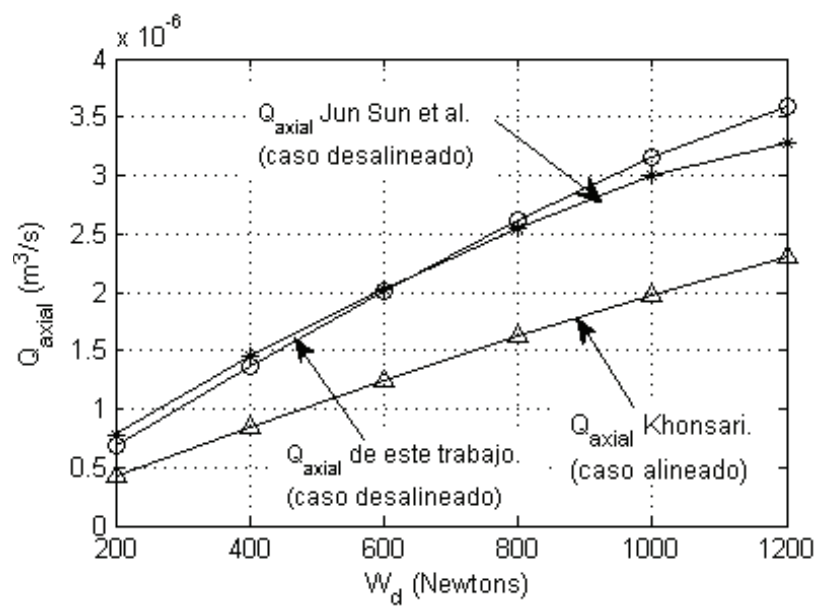

Figura 7. Comparación de $Q_{\text {axial }}$ con y sin desalineamiento

La figura 8 muestra la comparación de $F_{f} / W$ con desalineamiento obtenida por Jun et al. (2005) y la obtenida en este trabajo. Se puede ver que aunque los valores llevan la misma tendencia, los resultados de este trabajo son 1.4 veces (aproximadamente) más grandes que los reportados por Jun et al. (2005). También, los resultados sin desalineamiento por Khonsari y Booser (2001) son 1.4 veces (aproximadamente) más grandes que los resultados sin desalineamiento reportados por Jun et al. (2005). La figura 8 muestra que el incremento de carga $W_{d}$ causa un incremento muy pequeño en $F_{f} / W$ del caso desalineado con respecto al caso alineado. Por ejemplo, utilizando los valores dados por Jun et al. (2005) para $W_{d}=1000 \mathrm{~N}$ se tiene que $F_{f} / W$ se incrementa en 1.093 veces. Para la misma carga se tiene un incremento de de $F_{f} / W 1.11$ veces entre los valores de Khonsari y Booser (2001) (caso alineado) y los de este trabajo (caso desalineado). Utilizando los resultados de este trabajo se tiene que la fuerza de fricción viscosa $F_{f}=22.6 \mathrm{~N}$ para $W_{d}=200 \mathrm{~N}$ y $F_{f}=28.5 \mathrm{~N}$ para $W_{d}=1200 \mathrm{~N}$, notando un pequeño incremento en la fuerza de fricción. En resumen, el desalineamiento tiene poco efecto sobre la fuerza de fricción viscosa.

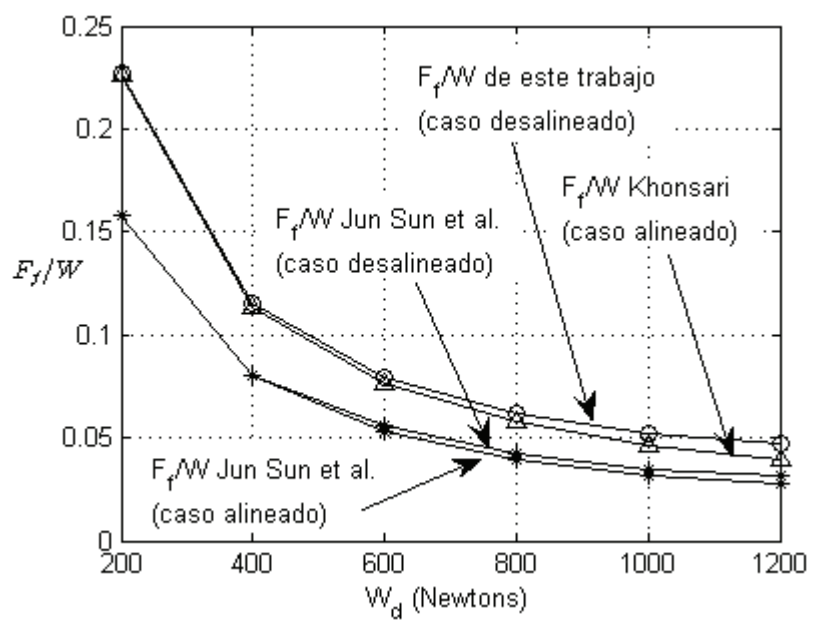

Figura 8. Comparación de $F_{f} / W$ con y sin desalineamiento

Las figuras 5, 6, 7 y 8 contienen resultados para un caso específico, los cuales aunque son de interés, no son aplicables a un caso de diseño que contenga diferentes parámetros de la chumacera, del eje, propiedades del lubricante y condiciones de operación. Los resultados de este trabajo se presentan en las siguientes figuras de forma adimensional para casos de diseño con relación de $L / D=1$ y diferentes valores de $\xi_{x}$.

La figura 9 muestra la relación entre el número de Sommerfeld y la excentricidad $\varepsilon_{0}$ (excentricidad del centro del muñón) para diferentes valores de desalineamiento $\xi_{x}$. Esta figura muestra que para un mismo número de Sommerfeld (mismas condiciones de opera- 
ción) la excentricidad $\varepsilon_{0}$ se reduce a medida que el desalineamiento $\xi_{x}$ aumenta. Por lo tanto, para determinar las condiciones de lubricación $\left(h_{\min n}, p_{\max }, Q_{\text {axial }}\right.$ y $\left.(R / C) f\right)$ será necesario determinar primero el punto de equilibro $\varepsilon_{0}$ del centro del muñón. Por ejemplo, si para determinadas condiciones de operación se tuviese un número de Sommerfeld de 0.3 entonces para el caso alineado $\varepsilon_{0}$ sería igual a 0.37 y para un caso desalineado con $\xi_{x}=0.7 \mathrm{el}$ punto de equilibrio $\varepsilon_{0}$ estaría en 0.30 aproximadamente (figura 9). La tabla 1 muestra las condiciones de operación que se obtienen de las figuras 10, 11, 12 y 13 para ambos casos.

Se puede ver en la figura 10 y la tabla 1 que el espesor de película $h_{\min }$ disminuye al aumentar el desalineamiento. También se observa en las figuras 11 y 12, y la tabla 1 que la presión máxima y el flujo axial se incre-

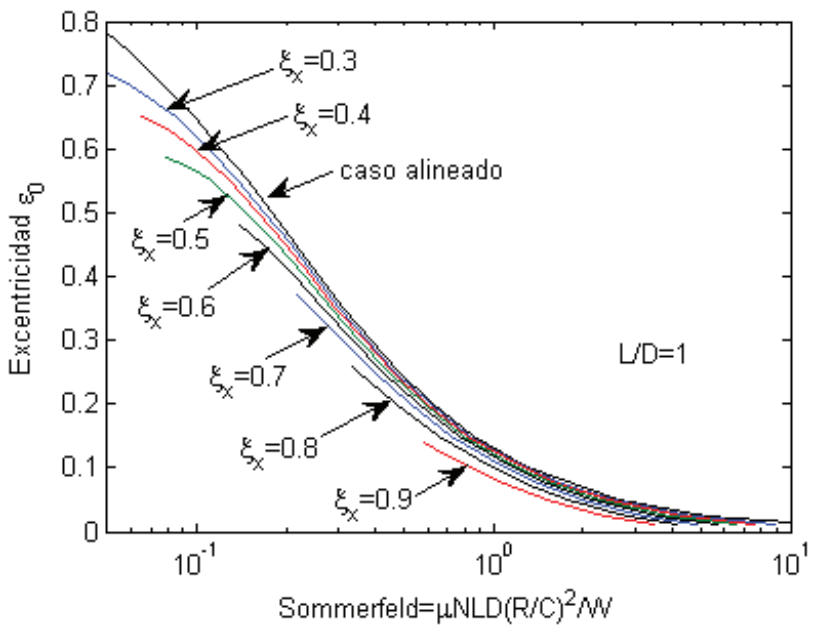

Figura 9. Excentricidad $\varepsilon_{0}$ contra número de Sommerfeld

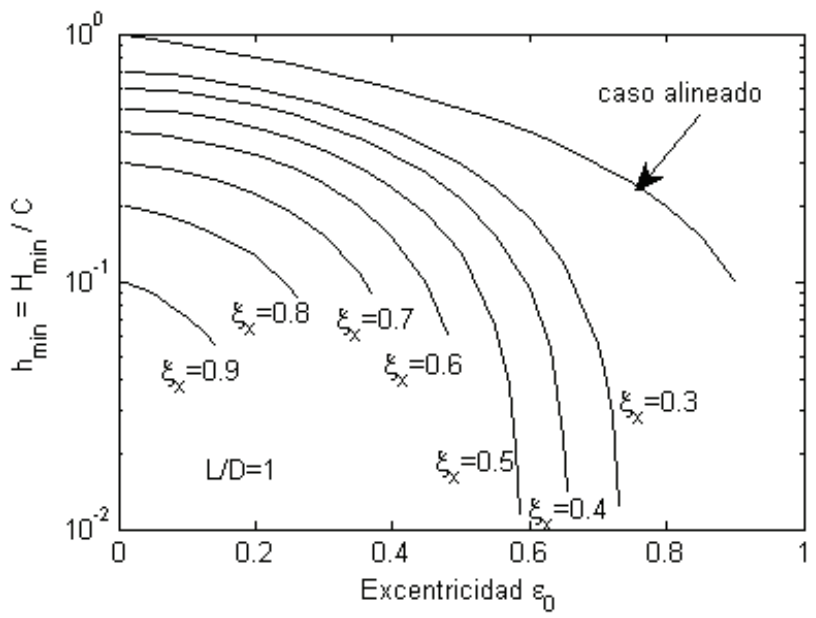

Figura 10. Espesor de película para diferentes valores de $\xi_{X}$ y $L / D=1$ mentan al considerar el desalineamiento. Al observar la figura 13 se podría interpretar que el coeficiente de fricción adimensional $(R / C) f$ disminuye al incrementarse el desalineamiento, lo que sería erróneo. La tabla 1 muestra el incremento de $(R / C) f$ al incrementarse $\xi_{x \prime}$ esto se debe a que $\varepsilon_{0}$ cambia de 0.37 del caso alineado a 0.30 para el caso con desalineamiento. Las figuras 10, 11,12 y 13 requieren la figura 9 para su correcta interpretación y uso. Es importante notar en la figura 9 que el desalineamiento ubica el centro del eje en una nueva posición de equilibrio.

Tabla I: Comparación de condiciones de operación para Sommerfeld 0.3 y $\mathrm{L} / \mathrm{D}=1$

\begin{tabular}{ccccc}
\hline Condición $\xi_{X}$ & $h_{\min }$ & $p_{\max } / \mu N(R / C)^{2}$ & $Q_{\text {axial }} / N R L C$ & $(R / C) f$ \\
\hline$\xi_{X}=0$ & 0.64 & 6.6 & 1.93 & 6.54 \\
$\xi_{X}=0.7$ & 0.15 & 12.6 & 2.3 & 7.3 \\
\hline
\end{tabular}

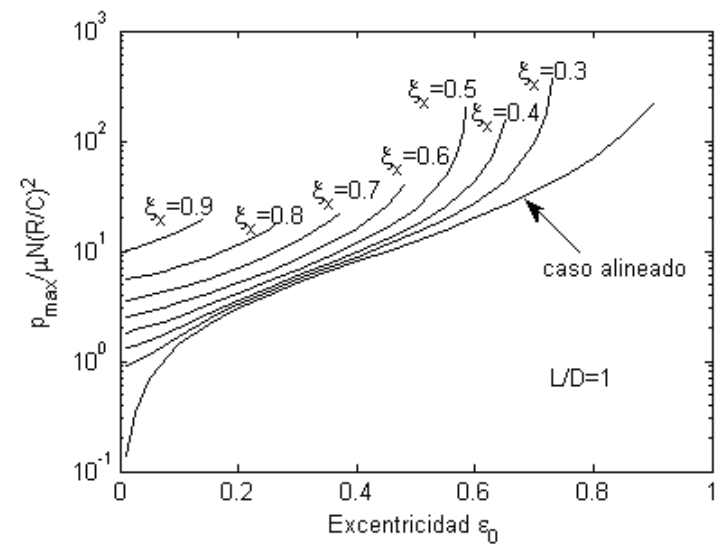

Figura 11. Presión máxima para diferentes valores de $\xi_{X}$ y $L / D=1$

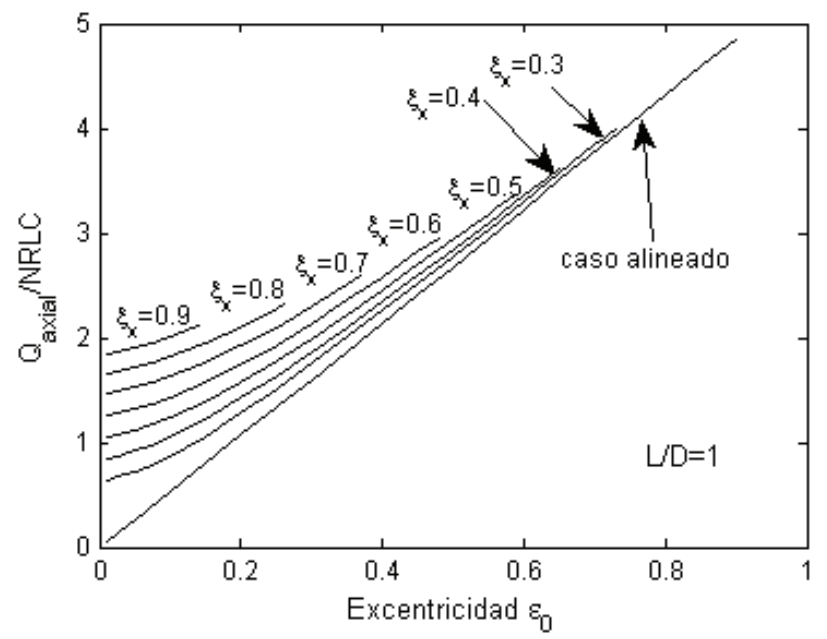

Figura 12. Flujo axial en función de $\xi_{X}$ y $L / D=1$ 


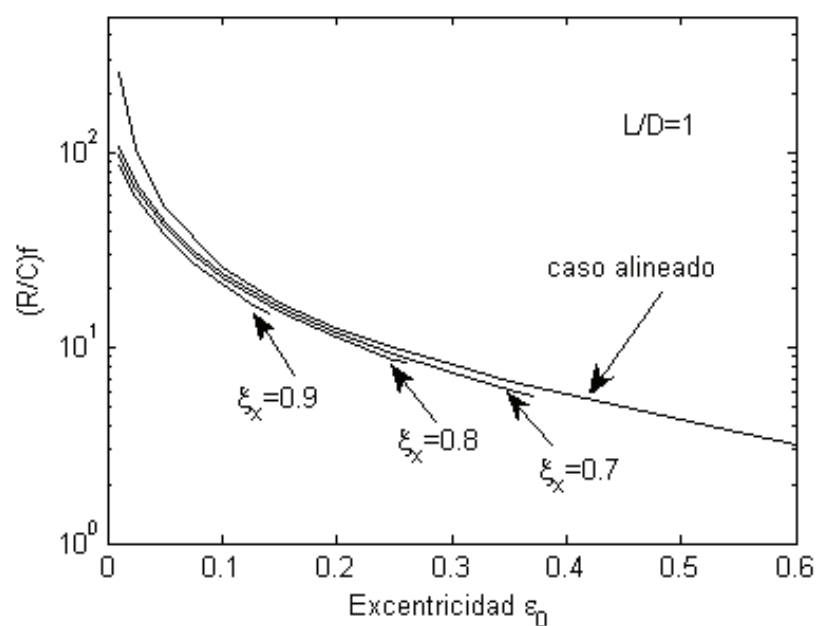

Figura 13. Fricción viscosa para $\xi_{\mathrm{x}}=0$ y $\xi_{\mathrm{x}}=0.9$ y para $\mathrm{L} / \mathrm{D}=1$

\section{Conclusiones}

En este trabajo se presenta la solución numérica de la ecuación de Reynolds que considera el desalineamiento del munón en la chumacera debido al peso y a la flexibilidad del rotor. Se emplea una expresión modificada del espesor de película que incluye el desalineamiento angular del eje por el efecto del peso del rotor.

El ángulo de desalineamiento $\alpha_{x}$ se calcula por medio de la teoría de vigas y se presenta una expresión para calcular el máximo ángulo de desalineamiento $\alpha_{\max }$ permitido por la geometría de la chumacera. Los resultados de las condiciones de lubricación con desalineamiento de $h_{\text {minn }}, p_{\text {max }}$, y $Q_{a x a l}$ y $(R / C) f$ que se obtienen en este trabajo se comparan con resultados reportados en la literatura, obteniéndose una buena aproximación. También se hace la comparación de los resultados de las condiciones de lubricación con desalineamiento que se obtienen en este trabajo y resultados sin desalineamiento reportados en la literatura, lo que muestra la importancia de considerar el desalineamiento en el diseño de chumaceras hidrodinámicas. Se puede ver que al considerar el desalineamiento, el espesor de película $h_{\text {min }}$ disminuye y la presión máxima y el flujo axial se incrementan. Por su parte, la fricción viscosa tiene cambios moderados con incrementos del desalineamiento. También se muestra que la excentricidad del centro del muñón $\varepsilon_{0}$ se ubica en una nueva posición al aumentar el desalineamiento para las mismas condiciones de operación (mismo número de Sommerfeld) lo que debe de tomarse en cuenta para calcular las condiciones de lubricación hidrodinámica.

Los resultados de este trabajo se presentan en forma adimensional para un diseño preliminar de chumace- ras hidrodinámicas para $L / D=1$ y diferentes valores de desalineamiento angular.

\section{Agradecimientos}

Se agradece a la Universidad Tecnológica de la Mixteca por el tiempo asignado para la realización de este trabajo.

\section{Referencias}

Buckholz R.H., Lin J.F. The Effect of Journal Bearing Misalignment on Load and Cavitation for Non-Newtonian Lubricants. ASME Journal of Tribology, volumen 108 (número 4), octubre 1986: 645-654.

Childs D. Tubomachinery Rotordynamics: Phenomena, Modeling and Analysis, John Wiley \& Sons, Inc., 1993.

Dubois G., Ockvirk F., Wehe R. Experimental Investigation of Misaligned Couples and Eccentricity at Ends of Misaligned Plain Bearings, Tech note 3352, NACA, 1955.

Galleti-Manacorda L., Capriz G.Torque Produced by Misalignment in Short Lubricated Bearings. ASME Journal of Basic Engineering, volumen 65 (número 9), 1965.

Gere J.M., Timoshenko S.P. Mecánica de materiales, International Thomson Editores, 1998.

Guha S.K. Analysis of the Steady-State Characteristics of Misaligned Hydrodynamic Journal Bearing with Isotropic Roughness Effect. Tribology International, volumen 33 (número 1), 2000: 1-12.

Jun S., Changlin G., Zhiyuan L. An Experimental Study of Journal Bearing Lubrication Effected by Journal Misalignment as a Result of Shaft Deformation Under Load. ASME Journal of Tribology, volumen 127 (número 4), 2005: 813-819.

Jun S., Mei D., Yonghong F., Changlin G. Thermohydrodynamic Lubrication Analysis of Misaligned Journal Bearing with Rough Surface. ASME Journal of Tribology, volumen 132 (número 1), 2010: 011704.

Khonsari M.M. y Booser E.R. Applied Tribology: Bearing Design and Lubrication, John Wiley and Sons, Inc., 2001.

Lund J.W., Thomsen K.K. A Calculation Method and Data for the Dynamic Coefficients of Oil-Lubricated Journal Bearings, Topics in Fluid Journal Bearing and Rotor Bearing System. ASME, 1978: 1-28.

McKee S.A., McKee T.R. Pressure Distribution in Oil Film of Journal Bearings. Trans. Am. Soc. Mech. Engrs., (número 107), 1932: 149-165.

Mokhtar M.O.A., Safar Z.S., Abd-El-Rahman M.A.M. And Adiabatic Solution of Misaligned Journal Bearings. ASME Journal of tribology, volumen 107 (número 2), abril 1985: 263-267.

Nicholas D. Les Paliers Hydrodynamiques Sounis un Torseur de Forcesquelconques, (PhD thesis), INSA de Lyon, 1972.

Pierre I., Bouyer J., Fillon M. Thermohydrodynamic Behavior of Misaligned Plain Journal Bearings: Theoretical and Experi- 
mental Approaches. Tribology Transactions, volumen 47 (número 4), 2004: 594-604.

Qiu Z.L., Tieu A.K. Experimental Study of Freely Alignable Journal Bearing-Part I: Static Characteristics. ASME Journal of Tribology, volumen 118 (número 3), 1996: 498-502.

Raimondi A.A., Boid J.A. Solution for the Finite Journal Bearing and its Application to Analysis and Design. ASLE Transactions, volumen 1 (número 1), 1958: 159-209.

Szeri A.Z. Fluid Film Lubrication: Theory and Design, Cambridge University Press, 1998.

\begin{abstract}
Este artículo se cita:
Citación Chicago

Antonio-García, Alberto, Jesús Linares-Flores, Manuel Arias-Montiel. Investigación numérica de las condiciones de lubricación en chumaceras hidrodinámicas con el efecto del desalineamiento del eje. Ingeniería Investigación y Tecnología XIV, 01 (2013): 89-98.
\end{abstract}

\section{Citación ISO 690}

Antonio-García A., Linares-Flores J., Arias-Montiel M. Investigación numérica de las condiciones de lubricación en chumaceras hidrodinámicas con el efecto del desalineamiento del eje. Ingeniería Investigación y Tecnología, volumen XIV (número 1), eneromarzo 2013: 89-98.

\section{Semblanza de los autores}

Alberto Antonio-García. Obtuvo el grado de doctor en ciencias en ingeniería mecánica en la Sección de Estudios de Posgrado e Investigación del Instituto Politécnico Nacional (ESIME-IPN). Su tesis sobre el cálculo de coeficientes rotodinámicos de chumaceras hidrodinámicas fue reconocida por el IIE y la CFE con el segundo lugar en los XXIII Certámenes Nacionales de Tesis 20052006, en la disciplina de generación de energía eléctrica en el nivel doctorado. Actualmente es profesor investigador de la carrera de ingeniería en mecatrónica en la Universidad Tecnológica de la Mixteca.

Jesús Linares-Flores. Realizó sus estudios de licenciatura en electrónica en la BUAP, posteriormente realizó sus estudios de maestría en ciencias en la UDLA-PUE con la especialidad en electrónica de potencia. En 2006 recibió el grado de doctor en ciencias, otorgado por el Cinvestav-IPN en el Departamento de Ingeniería Eléctrica, sección mecatrónica, desde el año 2006 pertenece al SNI. Actualmente es director del Instituto de Electrónica y Mecatrónica de la Universidad Tecnológica de la Mixteca.

Manuel Arias-Montiel. Estudió la licenciatura en ingeniería mecánica en la Universidad Autónoma Metropolitana en 2002. En 2005 recibió el título de maestro en ciencias en ingeniería eléctrica por parte del Centro de Investigación y de Estudios Avanzados del IPN y en 2010 el grado de doctor en ciencias en ingeniería eléctrica por parte de la misma institución. De 2005 a 2010 laboró como profesor de cátedra en el Tecnológico de Monterrey Campus Estado de México y actualmente es profesor investigador en el Instituto de Electrónica y Mecatrónica de la Universidad Tecnológica de la Mixteca. 